\title{
ALTERAÇÕES AGUDAS DOS NÍVEIS DE NEUROTRANSMISSORES EM CORPO ESTRIADO DE RATOS JOVENS APÓS ESTADO EPILÉPTICO INDUZIDO POR PILOCARPINA
}

\author{
Rivelilson Mendes de Freitas', Francisca Cléa Florenço de Sousa², \\ Silvânia Maria Mendes Vasconcelos², Glauce Socorro Barros Viana², \\ Marta Maria de França Fonteles ${ }^{2,3}$
}

\begin{abstract}
RESUMO - Altas doses de agonista colinérgico muscarínico, pilocarpina, produzem alterações comportamentais, convulsões e estado epiléptico em ratos. 0 objetivo desse estudo foi verificar as alterações nas concentrações dos neurotransmissores em corpo estriado de ratos em desenvolvimento após estado epiléptico induzido pela pilocarpina. Ratas Wistar foram tratadas com uma única dose de pilocarpina ( $400 \mathrm{mg} / \mathrm{Kg}$; s.c.). Controles receberam salina. A concentração dos neurotransmissores foi determinada através do HPLC, no corpo estriado de ratos que no período de observação de 1 e $24 \mathrm{~h}$ desencadearam estado epiléptico e não sobreviveram à fase aguda do quadro convulsivo. Foi observada redução nos níveis de dopamina, serotonina, ácido dihidroxifenilacético, ácido 5-hidroxiindolacético, e aumento no ácido 4-hidroxi-3-metoxi-fenilacético. Os resultados mostraram que a ativação do sistema colinérgico pode interagir com os sistemas dopaminérgico e serotonérgico nos mecanismos referentes à fase aguda do processo convulsivo.
\end{abstract}

PALAVRAS-CHAVE: dopamina, serotonina, convulsão, pilocarpina, corpo estriado.

\begin{abstract}
Acute alterations of neurotransmitters levels in striatum of young rat after pilocarpine-induced status epilepticus

ABSTRACT - High doses of the muscarinic cholinergic agonist, pilocarpine, result in behavioural changes, seizures and status epilepticus in rats. The purpose of the present work is to invetigate the striatal neurotransmissors level in young rats after status epilepticus induced by pilocarpine. Wistar rats were treated with a single dose of pilocarpine $(400 \mathrm{mg} / \mathrm{Kg}$; s.c.). Controls received saline. Young animals were closed observed for behavioural changes during 1 and $24 \mathrm{~h}$. In these periods, the animals that developed status epilepticus and didn't survive this acute phase of seizures had the brains removed and striatal neurotransmissors level determined by HPLC. The concentration of dopamine, serotonine, dihydroxyphenylacetic acid, 5hydroxyindolacetic acid was reduced and an increase in 4-hydroxy-3-methoxy-phenylacetic acid was observed. These results suggest that cholinergic activation can interage with dopaminergic and serotonergic systems in acute phase of the convulsive process in immature striatum.
\end{abstract}

KEY WORDS: dopamine, serotonine, seizures, pilocarpine, striatum.

Agonistas muscarínicos administrados em altas doses induzem a produção de convulsões motoras límbicas e estado epiléptico em ratos adultos ${ }^{1,2}$. 0 modelo de convulsão com dose alta de pilocarpina $(400 \mathrm{mg} / \mathrm{Kg})$ revela o processo convulsivo através de alterações comportamentais, eletroencefalográficas e pela presença de intensa lesão cerebral ${ }^{3-5}$, permitindo também o estudo das alterações em nível de receptores ${ }^{6}$, e/ou dos níveis das monoaminas e seus metabólitos ${ }^{7,8}$. As convulsões produzidas pelos agonistas colinérgicos em animais parecem depender da ativação do receptor muscarínico e do envolvimento dos fosfoinositídios ${ }^{4,6}$, sugerindo ações pós sinápticas. Vários estudos ${ }^{4,6}$, porém, indicam aumento não somente na atividade colinérgica pós-sináptica, mas na pré-sináptica também. Contudo, coDepartamento de Fisiologia e Farmacologia da Universidade Federal do Ceará (UFC), Fortaleza CE, Brasil: 'Mestrando em Farmacologia;
'Laboratório de Neurofarmacologia; ${ }^{3}$ Departamento de Farmácia.

Recebido 24 Setembro 2002, recebido na forma final 19 Dezembro 2002. Aceito 14 Janeiro 2003.

Dra. Marta Maria de França Fonteles - Departamento de Farmácia e Laboratório de Neurofarmacologia do Departamento de Fisiologia e Farmacologia da UFC - Rua Cônsul Gouveia 469 - 60335-390 Fortaleza CE - Brasil. FAX: 85 288 8257. E-mail: martafonteles@hotmail.com 
mo esses achados podem contribuir para explicar a origem e o mecanismo de todo o processo convulsivo, ainda precisam ser melhor determinados.

Para esse fim, vários aspectos têm sido observados, a saber: a interferência do sistema de neurotransmissão muscarínico ${ }^{6}$, dopaminérgico ${ }^{9,10}$, glutamatérgico ${ }^{11,12}$, e GABAérgico ${ }^{13-15}$, e o estudo dos segundos mensageiros ${ }^{6}$ nas convulsões utilizando pilocarpina $400 \mathrm{mg} / \mathrm{Kg}^{13}$. Tem sido feita outra abordagem que favorece o conhecimento da epilepsia em relação à idade: o estudo das convulsões em animais na fase de desenvolvimento ${ }^{16}$, uma vez que 0 cérebro imaturo, com suas inervações e densidade de receptores ainda em desenvolvimento, pode ajudar na investigação da origem das convulsões, incluindo a relação entre a ativação do receptor e o processo de transdução de $\operatorname{sinal}^{16}$ e as possíveis alterações nas concentrações dos neurotransmissores ${ }^{15}$. Várias pesquisas ${ }^{16,17,18}$ têm sido desenvolvidas utilizando, em particular, animais de 21 dias de idade, no modelo de convulsão com pilocarpina $400 \mathrm{mg} / \mathrm{Kg}$. Foi relatado que os animais neonatos ${ }^{16}$ apresentam-se vulneráveis às convulsões induzidas por pilocarpina em altas doses e que a atividade convulsiva parece ter características diferentes em função da idade. 0 agonista colinérgico induz manifestações comportamentais e eletroencefalográficas de estado epiléptico em ratos a partir da segunda semana de vida, e essas alterações são semelhantes às da epilepsia do lobo temporal de humanos ${ }^{16,17}$.

Como o mecanismo das convulsões ainda não foi completamente esclarecido, decidiu-se investigar, através de estudos neuroquímicos, as alterações que poderiam ocorrer com 1 e $24 \mathrm{~h}$ sobre a concentração das monoaminas e metabólitos, após estado epiléptico induzido por pilocarpina $(400 \mathrm{mg} / \mathrm{Kg})$, em ratos jovens com 21 dias de idade.

\section{MÉTODO}

Um número total de 90 animais foi utilizado nos experimentos. Ratos Wistar jovens (21 dias de idade; fêmeas; $n=90$ ), com peso variando de 25 e $35 \mathrm{~g}$, provenientes do Biotério Central da Universidade Federal do Ceará, foram tratados com uma única dose de pilocarpina $(400 \mathrm{mg} / \mathrm{Kg}$; s.c.; $n=60$ ). Os animais foram divididos em 2 grupos com o mesmo número e observados durante 1 e $24 \mathrm{~h}$ após a administração. Os animais controles foram tratados com solução salina ( $\mathrm{NaCl}, 0,9 \%$; s.c.; $n=30$ ). Durante todos os experimentos, os animais foram mantidos em gaiolas no máximo com 6 animais, em condições ambientais semeIhantes, com ciclos de alternância claro/escuro de $12 \mathrm{~h}$, recebendo ração padrão tipo Purina e água ad libitum. Para o tratamento agudo com pilocarpina, a administração foi realizada com os animais no $21^{\circ}$ dia de vida. Os animais que apresentaram convulsão, estado epiléptico e não sobreviveram ao tratamento durante o período de 1 e $24 \mathrm{~h}$ de observação, foram decapitados e imediatamente o corpo estriado foi dissecado sobre gelo para preparação dos homogenatos utilizados na realização dos estudos neuroquímicos.

Os níveis cerebrais de monoaminas foram estudados em homogenatos a $10 \%$ preparados em tampão fosfato de sódio $(150 \mathrm{mM} ; \mathrm{pH} 7,4)$. Para a determinação dos neurotransmissores dopamina (DA), serotonina (5-HT) e seus metabólitos ácido dihidroxifenilacético (DOPAC), ácido 4hidroxi-3-metoxi-fenilacético (HVA), ácido 5-hidroxiindolacético (5-HIAA), foi utilizado um equipamento de HPLC da Shimadzu, modelo LCD-6 ${ }^{A}$, com detecção amperométrica. Para a separação das monoaminas, foi usada uma coluna de fase reversa (Shim-Pack CLC-ODS, $25 \mathrm{~cm}$ ). A fase móvel foi preparada com ácido cítrico monohidratado $150 \mathrm{mM}$, octil sulfato de sódio $67 \mathrm{mM}$, tetrahidrofurano $2 \%$, acetonitrila 45, utilizando água deionizada. $\mathrm{O} \mathrm{pH}$ da fase móvel foi ajustado para 3,0 com NaOH $10 \mathrm{mM}$. A quantificação dos picos obtidos foi feita com o auxílio de uma curva padrão. Valores absolutos foram corrigidos quanto à recuperação das cânulas e expressões de variação em relação aos valores basais.

Para estudo estatístico foi utilizada a análise de variância (ANOVA) para múltiplas comparações e o teste tStudent's como teste post hoc. As diferenças foram consideradas estatisticamente significativas quando $p<0,05$.

\section{RESULTADOS}

A Tabela 1 mostra que doses convulsivas de pilocarpina diminuíram de maneira significativa os níveis de dopamina (DA) de $64 \%$ em $1 \mathrm{~h}[\mathrm{~T}(10)=7,025$, $p<0,0001]$, e de $48 \%$ em $24 \mathrm{~h}[\mathrm{~T}(10)=5,789, \mathrm{p}<0,0002]$; do ácido dihidroxifenilacético (DOPAC) de $35 \%$ em $1 \mathrm{~h}$ $[\mathrm{T}(10)=3,575, \mathrm{p}<0,0051]$, e de $28 \%$ em $24 \mathrm{~h}[\mathrm{~T}(9)=$ $3,361, p<0,0084]$, e que aumentaram a concentração

Tabela 1. Efeitos da administração da pilocarpina $(400 \mathrm{mg} / \mathrm{Kg}$ ) sobre os níveis de DA, DOPAC e HVA em corpo estriado de ratos jovens.

\begin{tabular}{lccc}
\hline Monoaminas & Grupo & $1 \mathrm{~h}$ & $24 \mathrm{~h}$ \\
\hline DA & $\mathrm{C}$ & $3088 \pm 210(7)$ & $3088 \pm 210(7)$ \\
& $\mathrm{CV}$ & $1112 \pm 186^{*}(5)$ & $1614 \pm 143^{*}(5)$ \\
DOPAC & $\mathrm{C}$ & $861 \pm 37(7)$ & $861 \pm 37(7)$ \\
& $\mathrm{CV}$ & $563 \pm 74^{*}(5)$ & $623 \pm 63^{*}(5)$ \\
HVA & $\mathrm{C}$ & $523 \pm 19(7)$ & $523 \pm 19(7)$ \\
& $\mathrm{CV}$ & $1150 \pm 19^{*}(5)$ & $679 \pm 59^{*}(5)$ \\
\hline
\end{tabular}

Os animais com 21 dias de idade foram tratados com uma única dose de pilocarpina $(400 \mathrm{mg} / \mathrm{Kg}$; s.c.; $\mathrm{n}=5)$ e os controles com salina $0,9 \%(\mathrm{n}=7)$, sendo observados durante 1 e $24 \mathrm{~h}$ após a administração. Os valores são expressos como a média ( $\mathrm{ng} / \mathrm{g}$ de tecido) \pm EPM e o número de ratos sacrificados em cada grupo indicado entre parênteses. ${ }^{*} p<0.05$, controle versus Pilocarpina (ANOVA e teste t-Student post hoc). Abreviações: C (rato controle), CV (rato convulsivo), DA (dopamina) e seus metabólitos DOPAC (ácido dihidroxifenilacético), HVA (ácido 4-hidroxi-3-metoxi-fenilacético). 
Tabela 2. Efeitos da administração da pilocarpina (400 $\mathrm{mg} / \mathrm{Kg}$ ) sobre os níveis de 5-HT e 5-HIAA em corpo estriado de ratos jovens.

\begin{tabular}{lccc}
\hline Monoaminas & Grupo & $1 \mathrm{~h}$ & $24 \mathrm{~h}$ \\
\hline 5 5-HT & $\mathrm{C}$ & $848 \pm 161(7)$ & $848 \pm 161(7)$ \\
& $\mathrm{CV}$ & $191 \pm 48^{*}(5)$ & $360 \pm 46^{*}(5)$ \\
5 5-HIAA & $\mathrm{C}$ & $1304 \pm 201(7)$ & $1304 \pm 201(7)$ \\
& $\mathrm{CV}$ & $469 \pm 67^{*}(5)$ & $456 \pm 39 *(5)$ \\
\hline
\end{tabular}

Os animais com 21 dias de idade foram tratados com uma única dose de pilocarpina $(400 \mathrm{mg} / \mathrm{Kg}$; s.c.; $n=5)$ e os controles com salina $0,9 \%(n=7)$, sendo observados durante 1 e $24 \mathrm{~h}$ após a administração. Os valores são expressos como a média ( $\mathrm{ng} / \mathrm{g}$ de tecido) \pm EPM e o número de ratos sacrificados em cada grupo indicado entre parênteses. ${ }^{*} p<0.05$, controle versus Pilocarpina (ANOVA e teste t-Student post hoc). Abreviações: $\mathrm{C}$ (rato controle), CV (rato convulsivo), 5-HT (serotonina) e seu metabólito 5-HIAA (ácido 5-hidroxiindolacético).

do ácido 4-hidroxi-3-metoxi-fenilacético (HVA) em $119 \%$, em $1 \mathrm{~h}[\mathrm{~T}(8)=2,495, \mathrm{p}<0,0372]$, e em $30 \%$ no período de $24 \mathrm{~h},[\mathrm{~T}(9)=23,307, \mathrm{p}<0,0001]$.

Por sua vez, na Tabela 2, foi observada redução significativa da concentração da serotonina (5-HT) de $77 \%$ em $1 \mathrm{~h}[\mathrm{~T}(9)=4,226 ; \mathrm{p}<0,0022]$ e de $57 \%$ em $24 \mathrm{~h}[\mathrm{~T}(9)=3,149 ; \mathrm{p}<0,0118]$. Também houve redução nos valores do seu metabólito ácido 5-hidroxiindolacético (5-HIAA) em 64\% em $1 \mathrm{~h}[\mathrm{~T}(8)=3,924$; $p<0,0044]$, e 65\% em 24h [T(10) $=4,885 ; p<0,0006]$.

A Figura 1 mostra a comparação, em valores percentuais, percentagem das concentrações das monoaminas DA e 5-HT e dos metabólitos DOPAC, HVA e 5-HIAA em corpo estriado de ratos jovens durante os períodos de 1 e $24 \mathrm{~h}$ após estado epiléptico induzido por pilocarpina.

\section{DISCUSSÃO}

A pilocarpina promoveu a indução de convulsões que se desenvolveram para o estado epiléptico de forma grave e de longa duração. Poucos animais sobreviveram. Os animais que apresentaram convulsões, estado epiléptico e não sobreviveram até à primeira hora e às $24 \mathrm{~h}$ de observação, foram estudados quanto às prováveis modificações dos neurotransmissores, nessas condições. Foi vista redução, de forma rápida e drástica, nos níveis de DA, 5-HT, DOPAC, 5-HIAA, e concentração aumentada de HVA. Algumas pesquisas têm focalizado o efeito epileptogênico da administração de alta dose de pilocarpina durante a ontogênese em ratos, na intenção de determinar os efeitos de doses convulsivas nos animais em desenvolvimento ${ }^{16-18}$. Além disso, estes estudos investigam a origem e a propagação das convulsões no cérebro em desenvolvimento, a fim de esclarecer o mecanismo do processo convulsivo. No presente estudo foram utilizados ratos com 21 dias de idade submetidos ao modelo de convulsão com pilocarpina sozinha $(400 \mathrm{mg} / \mathrm{Kg})$.

Nossos resultados mostraram que o tratamento agudo com pilocarpina resultou em morte de $68 \%$

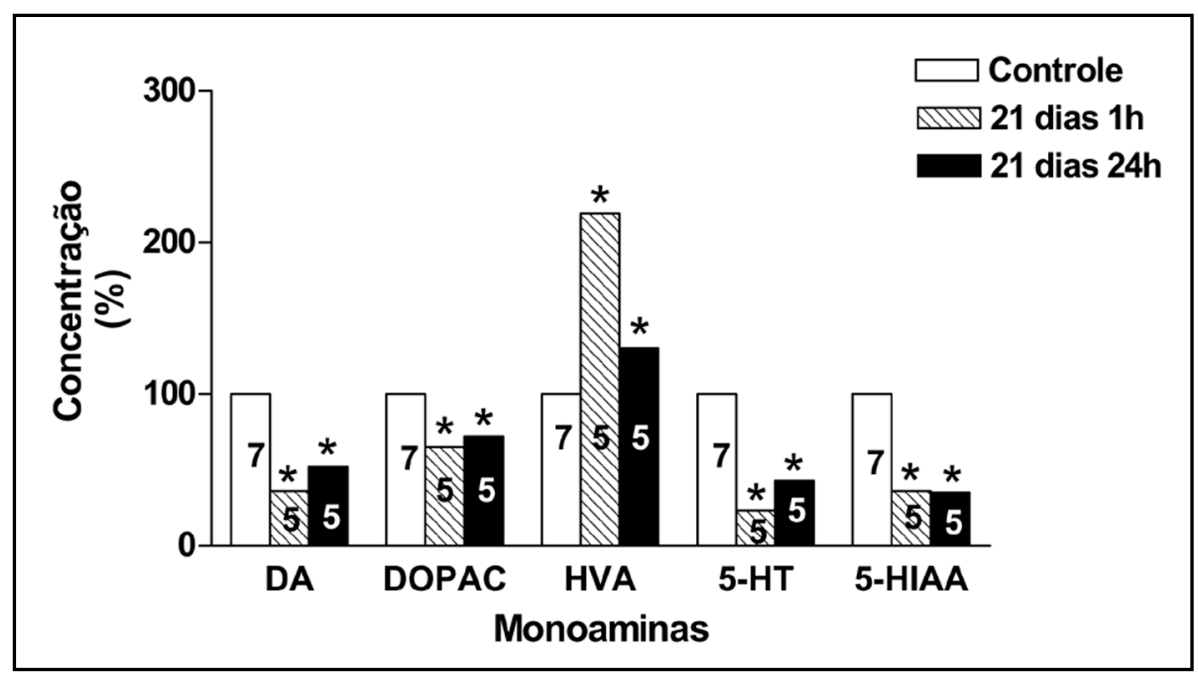

Fig 1. Percentagem da concentração das monoaminas em corpo estriado de ratos jovens após estado epiléptico induzido por pilocarpina $(400 \mathrm{mg} / \mathrm{Kg})$. Os animais com 21 dias de idade foram tratados com uma única dose de pilocarpina (400mg/Kg; s.c.; $n=5)$ e os controles com salina $0,9 \%(n=7)$, sendo observados durante 1 e $24 \mathrm{~h}$ após a administração. Os valores são expressos em percentagem (\%) e o número de ratos sacrificados em cada grupo inserido na barra. ${ }^{*} p<$ 0.05 , controle versus Pilocarpina (ANOVA e teste t-Student post hoc). Abreviações: $C$ (rato controle), CV (rato convulsivo), DA (dopamina), 5-HT (serotonina), DOPAC (ácido dihidroxifenilacético), HVA (ácido 4-hidroxi-3-metoxi-fenilacético) e 5-HIAA (ácido 5-hidroxiindolacético). 
dos animais durante $1 \mathrm{~h}$ e também com $24 \mathrm{~h}$ de observação, revelando um porcentual de ocorrência de morte semelhante nos animais jovens independentemente do tempo de observação. O mesmo fato parece não ocorrer nos ratos adultos, Alguns estudos têm sugerido, na fase aguda das convulsões, relação quase imediata da acetilcolina com outros sistemas de neurotransmissão (adrenérgico, dopaminérgico e serotonérgico), uma vez que têm sido observados efeitos rápidos nas concentrações de noradrenalina, dopamina e serotonina ${ }^{7,14}$. Nos ratos jovens, em particular, a influência da imaturidade na relação entre os sistemas pode promover ações semelhantes do sistema colinérgico na fase aguda das convulsões independentemente do tempo, limitando a participação de mecanismos atenuantes da ativação colinérgica, provavelmente existentes no cérebro maduro.

Como relatado anteriormente, os níveis de monoaminas e metabólitos durante o estado epiléptico foram alterados nos ratos com 21 dias, observando-se redução nos níveis de DA, DOPAC, 5-HT, e 5-HIAA. O metabólito da dopamina HVA teve sua concentração aumentada em ambos os períodos de observação. Estes dados corroboram, em parte, os de El-Etri et al., ${ }^{7}$ que encontraram, em ratos adultos convulsivos após 24h de observação, aumento nos níveis de DA e 5-HT e de seus metabólitos DOPAC, HVA e 5-HIAA. Entretanto, após $1 \mathrm{~h}$ de observação, os níveis de DA e 5-HT não se alteraram, mas seus metabólitos sofreram aumento significativo nas suas concentrações.

Nossos achados apontaram para uma redução nos neurotransmissores e metabólitos, exceto para o HVA, durante a fase aguda das convulsões, até as primeiras 24 horas, indicando que, após a ativação colinérgica sabidamente necessária para desencadear o processo ${ }^{17,18}$, os níveis dos neurotransmissores foram semelhantes.

Acreditamos que mais estudos devam ser feitos, em tempos variados após o desenvolvimento do estado epiléptico e em diferentes idades, para maior conhecimento dos sistemas de neurotransmissores envolvidos em todo processo convulsivo.

\section{REFERÊNCIAS}

1. Turski WA, Cavalheiro EA, Schwarz M, Czuczwar SJ, Kleinronk Z, Turski L. Limbic seizures produced by pilocarpine in rats: behavioural, eletroencephalographic and neuropathological study. Behav Brain Res 1983;9:315-336.

2. Turski L, Ikonomidou C, Turski WA, Bortolotto ZA, Cavalheiro EA. Cholinergic mechanisms and epileptogenesis: the seizures induced by pilocarpine: a novel experimental model of intractable epilepsy. Synapse 1989;3:154-171.

3. Clifford DB, Olney JW, Maniotis A, Collins RC, Zorumski CF. The functional anatomy and pathology of lithium-pilocarpine and highdose pilocarpine seizures. Neuroscience 1987;23:953-968.

4. Persinger MA, Bureau YRJ, Kostakos M, Peredery O, Falter H. Behaviours of rats with insidious multifocal brain damage induced by seizures following single peripheral injections of lithium and pilocarpine. Physiol Behav 1993;53:849-866.

5. Marinho MMF, Sousa FCF, Bruin VMS, Aguiar LMV, Pinho RSN, Viana GSB. Inhibitory action of a calcium channel blocker (nimodipine) on seizures and brain damage induced by pilocarpine and lithiumpilocarpine in rats. Neurosci Letters 1997;235:13-16.

6. Marinho MMF, Sousa FCF, Bruin VMS, Vale MR, Viana GSB. Effects of lithium, alone or associated with pilocarpine, on muscarinic and dopaminergic receptors and on phosphoinositide metabolism in rat hippocampus and striatum. Neurochem Internat 1998;33:299-306.

7. El-Etri MM, Eniis M, Jiang M, Shipley MT. Pilocarpine-induced convulsions in rats: evidence for muscarinic receptor-mediated activation of locus coeruleus and norepinephrine release in cholinolytic seizure development. Exper Neurol 1993;121:24-39.

8. Khan GM, Smolders I, Ebinger G, Michotte Y. Anticonvulsant effect and neurotransmitter modulation of focal and systemic 2chloroadenosine against the development of pilocarpine-inducd seizure. Neuropharmacology 2000;39:2418-2432.

9. Barone P, Palma V, Debartomolemeis A, et al. Dopamine $\mathrm{D}_{1}$ and $\mathrm{D}_{2}$ receptors mediate opposite functions in seizures induced by lithiumpilocarpine. Europ J Pharmacol 1991;195:157-162.

10. Al-Tajir G, Starr BS, Starr MS. Proconvulsant effect of SKF 38393 mediated by nigral $D_{1}$ receptors. Europ J Pharmacol 1990;162:245-251.

11. Fujikawa DG, Daniels AH, Kim JS. The competitive NMDA receptor antagonist CGP 40116 protects against status epilepticus-induced neuronal damage. Epilepsy Res 1994;17:207-219.

12. Fujikawa DG. Neuroprotective effect of ketamine administered after status epilepticus onset. Epilepsia 1995;36:186-195.

13. Costa-Lotufo LV, Fonteles MMF, Lima ISP, et al. Attenuating effects of melatonin on pilocarpine-induced seizures in rats. Comp Biochem Physiol 2002;131:521-529.

14. Fritschy JM, Kiener T, Bouilleret V, Loup F. GABAergic neurons and GABAa-receptors in temporal lobe epilepsy. Neurochem Internat 1999;34:435-445.

15. Erakovic V, Zupan G, Varljen J, Laginja J, Simonic A. Lithium plus pilocarpine induced status epilepticus-biochemical changes. Neurosci Res 2000;36:157-166.

16. Hirsch E, Baram TZ, Snead III OC. Ontogenic study of lithium-pilocarpine induced status epilepticus in rats. Brain Res 1992;583:120-126.

17. Cavalheiro EA, Delrio FS, Turski WA, Calderezzo Filho LS, Bortolotto ZAA, Turski L. The susceptiblity of rats to pilocarpine-induced seizures is age-dependent. Dev Brain Res 1987;37:43-58.

18. Cavalheiro EA, Fernandes MJS, Turski L, Naffah-Mazzacoratti MG. Neurochemical changes in the hippocampus of rats with spontaneous recurrent seizures. Epilepsy Res 1992;(Suppl)9:239-248. 